\title{
CONSTRUIR CIUDADES RESILIENTES ANTE DESASTRES Transformando la gestión de información en conocimiento para desarrollar territorios más resilientes
}

\section{BUILD DISASTER-RESILIENT CITIES \\ Transforming information management into knowledge to develop more resilient territories}

\author{
A. Betsy Moretta Macías \& B. Dalton Andrade Rodríguez
}

Universidad San Gregorio de Portoviejo, República del Ecuador

betsy marita@hotmaill.com

dandrade091980@gmail.com

\begin{abstract}
RESUMEN
El presente estudio tiene como objetivo analizar cómo se ha administrado la información de la gestión riesgo antes, durante y después de las emergencias en la República del Ecuador, para poder plantear un mecanismo que permita fortalecer la gestión operativa, el conocimiento científico, técnico y tecnológico; y, contribuya a la reducción de los riesgos de desastres y creación territorios más resilientes; para esto, se realizó una investigación cualitativa apoyada en revisión documental y grupos focales, donde se examinaron las posiciones discursivas de diversos expertos en la materia; llegando a la conclusión que el desconocimiento de la estructura de las ciudades originan territorios vulnerables. Por lo que, las ciudades necesitan una correcta articulación entre: la generación de información, gestión operativa del territorio y gestión del conocimiento permitiendo analizar el contexto territorial, crear escenarios, y ejecutar planes sostenibles que impulsen un nuevo paradigma colaborativo mediante una plataforma territorial tecnológica multinstitucional e multidisciplinaria.
\end{abstract}

Palabras clave: generación de conocimiento, planificación territorial, resiliencia, riesgo.

Bloque temático: ciudad y ambiente

Tema: riesgo, vulnerabilidade y resiliencia

\section{ABSTRACT}

The objective of this study is to analyze how risk management information has being managed before, during and after emergencies in the Republic of Ecuador, in order to propose a mechanism that allows for the strengthening of operational management, scientific, technical and technological knowledge, and contributes to disaster risk reduction and the creation of more resilient territories. To this end, qualitative research has been conducting; supported by documentary review and focus groups, where the discursive positions of various experts in the field were examining; reaching the conclusion that the lack of knowledge of the structure of cities originates vulnerable territories. Therefore, cities need a correct articulation between the generation of information, operational management of the territory and knowledge management allowing analyzing the 


\section{SÃOPAULO15 17 LISBOA $25 \sim 26$ JUN 2020}

territorial context, creating scenarios, and executing sustainable plans that promote a new collaborative paradigm through a multi-institutional and multidisciplinary technological territorial platform.

Keywords: knoweledge generation, resilience, risk, territorial planning.

Reserch line: city and environment

Topic: risk, vulnerability and resilience

\section{Introducción}

Los cambios en el planeta se producen a través de fenómenos naturales como: lluvias, sismos, sequias, entre otros, necesarios para mantener el equilibrio ecosistémico (Lopez, 2017); estos cambios se tornan peligrosos debido al crecimiento acelerado de la población, desertificación de suelos, contaminación de aguas, aumento de migración hacia las ciudades, asentamientos irregulares en áreas de riesgo, sobreexplotación de los recursos que en situaciones extremas desencadenan afectaciones severas, mismas que se agudizan por procesos inadecuados de gestión (Lampis, 2013); las ciudades consumen una gran parte del suministro energético mundial y son responsables del $70 \%$ de las emisiones de gases efecto invernadero, incrementándose en la actualidad el número de eventos relacionados con desastres naturales a nivel mundial (Onu-habitat, 2019).

La República del Ecuador que se encuentra situada en el Hemisferio Occidental; al noroeste de América del Sur, entre la latitud $01^{\circ} 28^{\prime} \mathrm{N}$ a $05^{\circ} 02^{\prime} \mathrm{S}$ y de longitud $75^{\circ} 11^{\prime} \mathrm{W}$ a $81^{\circ} 04^{\prime}$. (Instituto Geográfico Militar de la República del Ecuador, 2020), no se escapa de esta realidad. Este territorio se encuentra inscrito en el riesgo y la vulnerabilidad debido a sus características geográficas. Otra de las problemáticas son los fuertes fenómenos de distribución desigual de la riqueza; en suma, un modelo de desarrollo desequilibrado y complejo.

En tal contexto, los sucesos de desastres implican necesariamente conflictos y crisis sociales significativas. Sin embargo, lo que aplica al origen de los riesgos y desastres, opera también para la búsqueda de soluciones o cambios. En este sentido, la planificación territorial y la gestión de riesgos aparece como un campo privilegiado para impulsar procesos de cambio social que van mucho más allá de la contención o la respuesta, y que implican cambios hacia una auténtica resiliencia social, e incluso, una reconfiguración de relaciones sociales territoriales.

Partiendo desde la visión que los desastres están relacionados con las prácticas humanas inadecuadas, resultado de una mala planificación territorial y políticas de desarrollo local (Maskrey, Cardona, García, Lavell, Macías, Romero, \& Chaux, 1993); este análisis busca en diversos actores sociales implicados en el desarrollo local sostenible ampliar la visión de cada una de sus áreas encontrando la complementariedad entre sí para que la sociedad pueda asumir con flexibilidad diversas situaciones límites producto de desastres y sobreponerse a ellas; esta práctica se sustenta en las políticas de acuerdos internacionales como: Marco de SENDAI y UN-HABITAT.

Considerando lo antes expuesto, se establece como objetivo general analizar cómo se ha administrado la información de la gestión riesgo antes, durante y después de las emergencias en la República del Ecuador, para poder plantear un mecanismo que permita fortalecer la gestión operativa, el conocimiento científico, técnico y tecnológico; y, contribuya a la reducción de los riesgos de desastres y creación territorios más resilientes. 


\section{SÃOPAULO $15 \sim 17 \cdot$ LISBOA $25 \sim 26$ JUN 2020}

\section{Metodología}

La presente investigación es de tipo cualitativa y toma de referencia un análisis descriptivo bibliográfico relacionado con: los conceptos de riesgo, resiliencia y revisión de datos estadísticos oficiales del sistema de gestión de riesgo nacional; así como también la elaboración de grupos focales donde "por medio de un espacio de opinión, se puede captar el sentir, pensar y vivir de los individuos" (Hamui \& Varela, 2013, p.2); en estos grupos se indago la perspectiva de servidores públicos del área de planificación territorial y la reducción de riesgos de desastres y expertos investigadores de la materia de reducción de riesgos. La investigación realizada fue una revisión bibliográfica, documental y crítica, de los documentos seleccionados y juicios generales de los expertos, contrastada con la experiencia profesional.

En los grupos focales la "representatividad no alude a una representación proporcional, sino a una representación de posiciones discursivas" (Arteaga, 2015, párr. 11) por lo tanto para entender como se ha administrado la información de la gestión riesgo antes, durante y después de las emergencias se constituyeron dos grupos focales cada uno conformado por 5 personas, la selección fue de tipo estructural siendo el primer grupo compuesto por servidores públicos de los departamentos gubernamental que intervienen en el proceso de emergencia y reconstrucción de las ciudades; y, el segundo grupo por expertos en la materia que se dedican a la investigación. Mediante esta técnica se indago las diferentes discrepancias sobre el manejo de las emergencias y la capacidad de resiliencia de la República del Ecuador.

Es importante mencionar que toda la muestra pertenece a la República del Ecuador; por lo cual la información obtenida rescata el discurso respecto a las principales percepciones, sentidos y acciones que tienen lugar en el país, con el consentimiento libre, previo e informado de los participantes.

\section{Resultados y discusión}

Los resultados y discusión de esta investigación se centraran en el análisis conceptual de términos relacionados con la reducción de los riesgos de desastres y la resiliencia, análisis estadísticos generales nacionales e interpretación de las diferentes posiciones discursivas por medio de grupos focales:

\subsection{Análisis teórico}

El riesgo natural es "la posibilidad de que un territorio y la sociedad que lo habita pueda verse afectado por un fenómeno natural de rango extraordinario" (Carcedo y Cantos, 2002, p. 55), la República del Ecuador por sus características geográficas posee diversas zonas de riesgo, entre las principales amenazas que se encuentran en el territorio están sismos, inundaciones, deslizamientos, tsunamis y pandemias. Uno de los eventos más recientes que dejo graves afectaciones fue el terremoto del 16 de abril de 2016, mismo que fue considerado como catástrofe siendo este "un efecto perturbador que provoca sobre un territorio un episodio natural extraordinario y que a menudo supone perdidas de las vidas humanas. Si la consecuencia de dicho episodio natural alcanza una magnitud tal que ese territorio necesita ayuda externa, en alto grado se habla de desastre" (Carcedo y Cantos, 2002, p. 55).

Después del desastre se crea la emergencia que es: situación de peligro que requiere una acción inmediata (Real Academia de la Lengua Española, 2016). Por tal razón, cuando se produce un desastre se realizan trabajos inmediatos con el fin de mitigar los efectos que ocasiono lo acontecido.

El término "mitigar" no debe tomarse en el sentido coloquial de "aliviar", sino en el sentido muy concreto y específico que se le da en la administración de desastres: Mitigación equivale en este contexto a reducción de la vulnerabilidad. A eliminar o reducir en lo posible esa incapacidad de la comunidad para absorber, mediante 


\section{SÃOPAULO15 17 LISBOA $25 \sim 26$ JUN 2020

el autoajuste, los efectos de un determinado cambio en el ambiente. A reducir su impotencia frente al riesgo, ya sea éste de origen humano o natural. A hacerla más flexible, más autónoma, más dueña de su relación con el ambiente. La mitigación adquiere especial importancia cuando, como en el caso de los riesgos naturales, no le podemos decir que "no" al riesgo (Maskrey, 1993, p. 21).

La forma en cómo se enfrenta la reconstrucción ante un desastre depende de la capacidad de la población para poder afrontar y superar los daños que afectan la habitabilidad del territorio (Paton, \& Johnston, 2017); por tal razón la resiliencia urbana surge ahora como un concepto emergente, desarrollado principalmente en los ámbitos de la psicología o la ecología, para describir y explicar por qué algunas ciudades que padecieron un agudo declive consiguen revitalizar su economía, regenerar su tejido social y renovar sus espacios deteriorados (Méndez, 2012, p. 215).

En el aspecto urbano la resiliencia debe estar fuertemente relacionada como la capacidad que tienen las comunidades para resistir catástrofes, sustentándose esta preparación mediante una planificación que determine objetivos, indicadores, proyectos tanto de prevención como de actuación que tienden siempre a evolucionar de forma cíclica ante cualquier eventualidad, para que la ciudad siempre pueda superar cualquier problema sea natural o antrópico (Cutter, Burton, \& Emrich, 2010).

La clave que todas las ciudades deben de considerar es la planificación en función de la adaptación urbana a los desafíos del cambio climático siendo un ejercicio que permite anticiparse para reducir riesgos y, a la vez, promover bienestar; no es solamente un proceso para minimizar la vulnerabilidad, sino también para identificar formas de maximizar oportunidades permitiendo a las comunidades adaptarse a los diferentes escenarios que pudieran surgir. (Barton, 2009).

\subsection{Estadísticas nacionales extraídas del sistema de gestión de riesgo}

Para este acápite se analizaron los datos de pérdidas por desastre contenidos en el Inventario Nacional de Desastres del Ecuador entre los años 1970-2007 y el año 2016 donde se suscitó el terremoto del 16 de abril que afecto principalmente a la costa ecuatoriana, se seleccionaron estos periodos de tiempo debido a su mayor registro de incidentes.

En el periodo comprendido entre 1970 y el 2007 en la República del Ecuador se presentaron 4025 registros de pérdidas de desastres, referidos a los Cantones en los cuales se subdividen las 22 Provincias del país (Castro, M. Y Fernández, L.). Los datos se clasificaron de acuerdo con el tamaño del impacto en extremos, grandes y pequeños tal como se detalla en la siguiente tabla:

\begin{tabular}{|l|l|l|l|l|l|l|l|l|}
\hline \multicolumn{7}{|c|}{ Registros y pérdidas por impacto, extremo, mayor y pequeño } \\
\hline Impacto & Registros & $\%$ & Muertos & $\%$ & $\begin{array}{l}\text { Viviendas } \\
\text { destruidas }\end{array}$ & $\%$ & $\begin{array}{l}\text { Viviendas } \\
\text { afectadas }\end{array}$ & $\%$ \\
\hline Extremo & 11 & 0,27 & 903 & 23 & 2279 & 20 & 22063 & 54 \\
\hline Mayor & 56 & 1,39 & 747 & 29 & 4294 & 37 & 7138 & 17 \\
\hline Pequeño & 3958 & 98,34 & 2269 & 58 & 4966 & 43 & 11844 & 29 \\
\hline Total & 4025 & 100 & 3919 & 100 & 11539 & 100 & 41045 & 100 \\
\hline
\end{tabular}

Tabla. 01 Registro y pérdidas por impacto: Creado en base a la información de la Secretaria de Riesgo del Ecuador y a la Comisión Europea y Comunidad Andina (2008). 


\section{SÃOPAULO15 17 LISBOA $25 \sim 26$ JUN 2020

Las pérdidas acumuladas por miles de desastres representan la mayoría de impactos en términos de muertos (57\%) y viviendas destruidas (43\%). Las víctimas asociadas a registros de pequeño impacto suman cerca del doble que los muertos de gran impacto (Castro, M. Y Fernández, L., 2008). En lo que tiene relación a los registros de gran impacto según sus categorías se encuentran las siguientes cifras:

Registros de gran impacto según categorías

\begin{tabular}{|l|l|l|l|l|l|l|l|l|}
\hline \multicolumn{1}{|c|}{ Categoría } & Fichas & $\%$ & Muertos & $\%$ & $\begin{array}{l}\text { Viviendas } \\
\text { destruidas }\end{array}$ & $\%$ & $\begin{array}{l}\text { Viviendas } \\
\text { afectadas }\end{array}$ & $\%$ \\
\hline Hidrometeorológicos & 51 & 76 & 1096 & 66 & 4419 & 67 & 25645 & 88 \\
\hline Geológicos & 7 & 10 & 337 & 20 & 1954 & 30 & 3556 & 12 \\
\hline Antrópicos & 2 & 3 & 20 & 1 & 200 & 3 & - & 0 \\
\hline Epidemias y plagas & 7 & 10 & 197 & 12 & - & 0 & - & 0 \\
\hline Total & 67 & 100 & 1650 & 100 & 6573 & 100 & 29201 & 100 \\
\hline
\end{tabular}

Tabla. 02 Registro de gran impacto: Creado en base a la información de la Secretaria de Riesgo del Ecuador y a la Comisión Europea y Comunidad Andina (2008).

En términos de vidas humanas los mayores impactos asociados con eventos hidrometeorológicos están estrechamente relacionadas con los episodios El Niño, durante los años 1983, 1987 y 1992-1993, encontrándose las mayores concentraciones de pérdidas en la región costera seguida de la región Sierra, donde se concentra la población (Comisión Europea y Comunidad Andina, 2008). Lamentablemente no se tienen cifras exactas de los gastos realizados en la reconstrucción.

En cuanto al terremoto del 16 de abril de 2016 dejo aproximadamente 587 personas fallecidas, 155 personas perdidas, 7.015 heridos, 25.376 personas refugiadas en albergues colectivos, más de 1.125 edificios destruidos, más de 829 edificios afectados, incluyendo 281 escuelas y varias obras de infraestructura, como carreteras y puentes dañados (Secretaria de Gestión de Riesgos, 2016). El Gobierno Central debido a este evento se vio obligado a designar aproximadamente USD3344 millones de dólares para la reconstrucción (Comité de Reconstrucción, 2016).

Existe una clara relación entre el gran porcentaje de desastres con los fenómenos hidrometeorológicos (lluvias, inundaciones, avenidas torrenciales, deslizamientos), mismos que ocasionan graves pérdidas sobre todo humanas, sin embargo todos estos datos únicamente se archivan y no se utilizan para generar nuevos conocimientos, escenarios y planes de prevención, entonces el gobierno debería de aprender de todos estos sucesos e invertir de su presupuesto anual un poco más prevención antes que lamentar perdidas y realizar gastos excesivos en reconstrucción; según el Laboratorio Internacional de Reducción de Riesgos de Desastres de Cambrige por cada dólar invertido en prevención se ahorra siete dólares en procesos de recuperación.

De las poblaciones susceptibles se podrían utilizar diversas variables como instrumento de conocimiento para fortalecer medidas de planificación de usos del suelo y obras de infraestructura como dotación de redes de alcantarillado (Comisión Europea y Comunidad Andina, 2008), pero para esto se necesita un trabajo de coordinación entre la academia, los técnicos de diferentes ramas, personal gubernamental, sociedad civil, que trabajen en conjunto para buscar mecanismos que reduzcan los riesgos y creen ciudades resilientes. Toda vez que como se demuestran en estas cifras la República del Ecuador esta susceptible a diferentes eventos por su ubicación geográfica, teniendo en consideración que todas las cifras antes mencionadas se pueden 


\section{XII $\square$ SÃOPAULO15 17 LISBOA $25 \sim 26$ JUN 2020

incrementar, debido al cambio climático que tiene un impacto global; un claro ejemplo de esto es que a nivel mundial 3.900 millones de personas, o aproximadamente la mitad de la población mundial, en el 2018 eran potencialmente expuestos a desastres naturales. Al contar si una región se vio afectada por desastres múltiples, este número salta a 10.7 mil millones de personas. (Centre for Research on the Epidemiology of Disasters, 2018); en un periodo de 9 años desde el 2008 hasta el 2017 se han producido 348 eventos en 10 países y solo en el 2018 se produjeron 315 eventos; estos eventos que trajeron consigo en el periodo del 2008 al 2017 la pérdida de 67,572 personas aproximadamente y solo en el 2018 la pérdida de 11,804 personas aproximadamente (Centre for Research on the Epidemiology of Disasters, 2018).

\subsection{Análisis de las diferentes posiciones discursivas por medio de grupos focales.}

En el grupo focal donde participaron los expertos, existe la percepción de que en el país no se sabe qué pasos seguir para poder hacer frente a las diversas problemáticas territoriales, que sobretodo dejan poblaciones fracturadas con escasa capacidad de recuperación.

La percepción de estos expertos es que no existe un correcto flujo en la administración de la información de la gestión riesgo antes, durante y después de las emergencias, ya que solamente son datos estadísticos que se archivan y en algunos casos no se encuentran ni actualizados o ni siquiera se monitorean los cambios que se producen en el ecosistema. Además recalcan la importancia de tener una matriz actualizada de información donde se midan diferentes aspecto como temperatura, precipitaciones, escorrentías de los ríos, gases contaminantes y también se ingrese información sobre los usos de suelo y diferentes dinámicas urbanas; mismas que puedan ser utilizadas para generar conocimiento, escenarios ayudando a prevenir y preparar a la población.

El grupo focal de las personas que laboran en la gestión de riesgo, se establece que este es un trabajo que necesita complementarse con diferentes áreas, como la academia, investigadores externos, gobiernos locales que permitan en conjunto trabajar para reducir los riesgos que amenazan a diferentes comunidades, debido a que no existe una partida presupuestaria a gran escala que les ayude a ser frente a esta problemática de manera autónoma.

Teniendo en consideración este contexto, los participantes de los grupos focales consideran que el Gobierno Nacional y los Gobiernos locales deben de reflexionar sobre las políticas de actuación para el desarrollo y la planificación territorial, desde la premisa que es mejor invertir en prevenir que en reconstruir, gestionando apoyo logístico con expertos y organismos ya sea nacionales o internacionales.

Entonces, debemos invertir en mitigar no desde el sentido coloquial de aliviar, sino en el sentido muy concreto y específico que se le da en la administración de desastres que equivale a reducir la vulnerabilidad, a eliminar en lo posible esa incapacidad de la comunidad para absorber, mediante el autoajuste, los efectos de un determinado cambio en el ambiente, a entender que no le podemos decir que "no" al riesgo (Maskrey, 1993).

Todos los aspectos antes mencionados deben considerarse para poder construir ciudades más resilientes. La forma en cómo se hace frente a la reconstrucción ante un desastre depende de la capacidad social para poder afrontar y superar los daños que pudieran afectar el territorio (Paton, \& Johnston, 2017); por lo que la resiliencia urbana surge como un concepto emergente, que trata de describir y explicar por qué algunas ciudades que padecieron un agudo declive consiguen revitalizar su economía, regenerar su tejido social y renovar sus espacios deteriorados (Méndez, 2012, p. 215).

En el aspecto urbano la resiliencia debe relacionarse como la capacidad que tienen la comunidad para resistir catástrofes, realizando análisis multidisciplinarios del territorio, creando escenarios que permita prepararse 


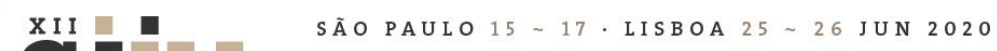

mediante una planificación objetiva que involucre diversas aristas e indicadores y proyectos tanto de prevención como de actuación que tienden siempre a evolucionar, para que la ciudad pueda superar de mejor manera cualquier problemática sea esta natural o antrópica (Cutter, Burton, \& Emrich, 2010).

Para poder realizar estos análisis multidisciplinarios, hay que recordar que la ciudad se configura como una estructura que puede analizarse por medio de sistemas urbanos, mismos que se articulan entre sí a modo de red, permitiendo el correcto funcionamiento de la ciudad. Si uno falla afecta en diferente medidas la cotidianidad del desarrollo de las ciudades. (Miranda \& Cornejo, 2010).

\subsection{Propuesta}

Considerando las problemáticas antes expuestas; $y$, las enseñanzas que dejaron los desastres ocurridos en la República del Ecuador, mediante los grupos focales se considera que la mejor alternativa para construir ciudades resilientes, es el conocimiento de la estructura social, económica y espacial de los territorios que permite mediante un análisis constante saber cuál es el estado actual para proponer políticas y generar planes que impulsen el desarrollo sostenible de las ciudades ya sea a corto o largo plazo.

Por tal razón, se plantea la creación de una plataforma tecnológica ya sea por cada ciudad o formando mancomunidades dependiendo de la extensión, el número de habitantes y la capacidad económica de cada territorio. Esta plataforma tendrá como objetivo fortalecer la gestión operativa, el conocimiento científico, técnico y tecnológico para la reducción de riesgos de desastres, integrando datos de cada ente científico, académico e instituciones públicas y privadas para tomar decisiones asociadas a diferentes variables de riesgo generando escenarios y análisis por medio de sistemas urbanos e incorporándolos a la planificación territorial y al marco normativo vigente.

\section{ESQUEMA GENERAL DE LA PLATAFORMA}
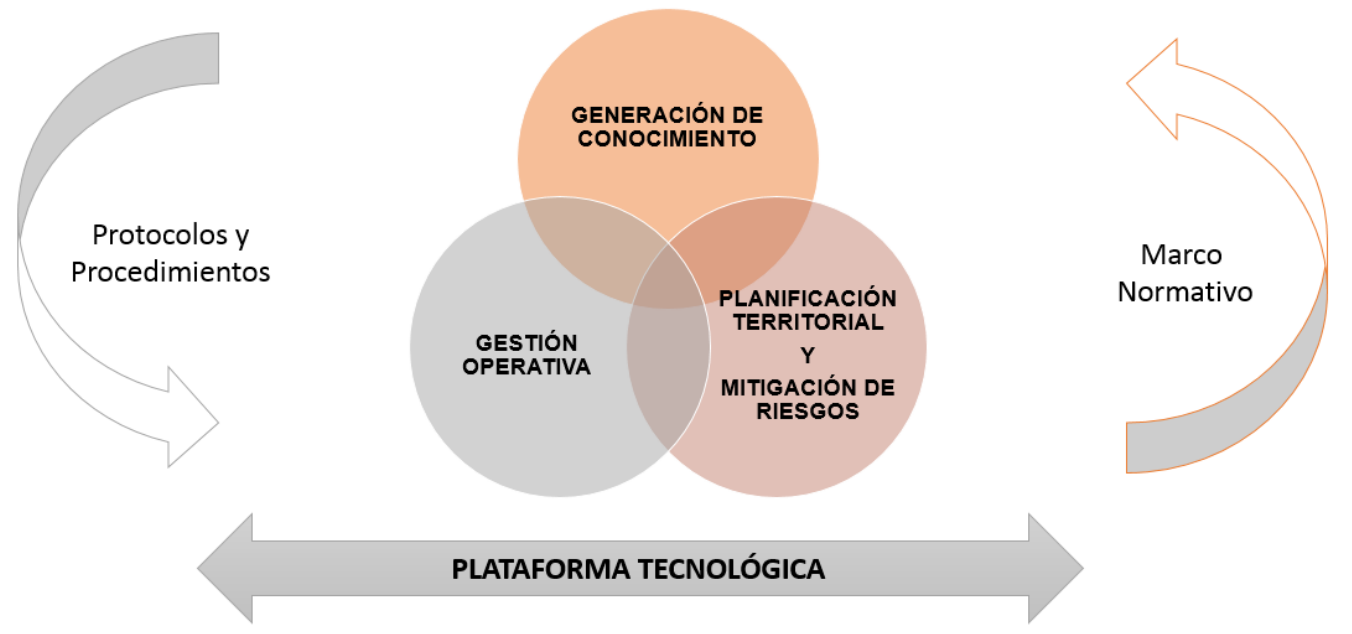

Fig. 01 Esquema general de la plataforma técnologica. Elaboración propia.

Dentro de la estructura orgánica funcional de la plataforma se encontrará una directiva que es la encargará del monitoreo, generación de estrategias, políticas y administración de la plataforma; se contará también con un comité consultivo que aportará de forma externa con el conocimiento científico para generar información (este comité lo conformará la academia, científicos e investigadores); un área de gestión operativa que articulará las acciones de respuesta interinstitucional antes, durante y después de una emergencia o incidente; $y$, un área 


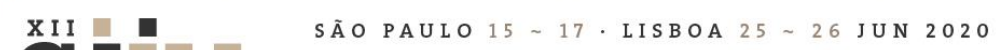

Seminário Internacional de Investigação em Urbanismo

Seminario Internacional de Investigación en Urbanismo

de planificación territorial y reducción de riesgos de desastre que tendrá como misión pensar el territorio para reducir vulnerabilidades.

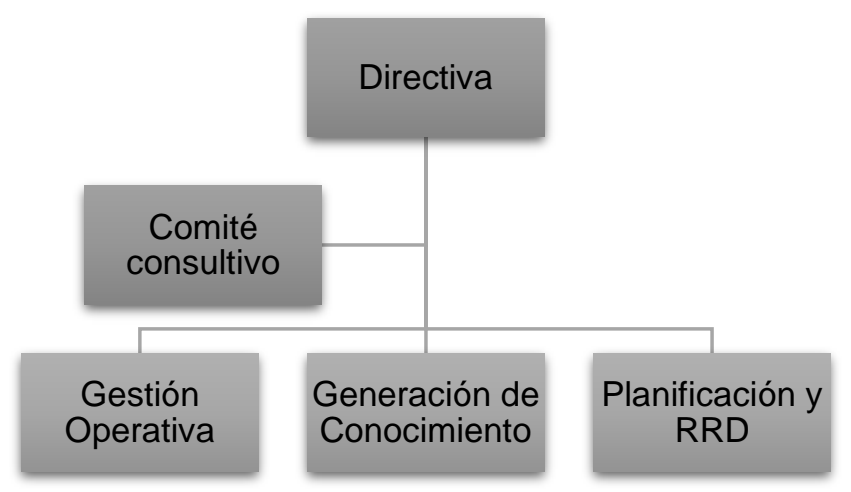

Fig. 02 Estructura organica funcional de la plataforma. Elaboración propia.

Esta plataforma debe de ser multidisciplinaria y multinstitucional donde la participación de los diferentes agentes sociales sea el soporte de toda la información y planes que se puedan generar y articular; entre los actores claves se encontrarían: gobiernos nacionales y locales, instituciones de respuesta estatales, instituciones de respuestas locales, sociedad civil, ONG, organismos de cooperación y financiamiento, academia, científicos, investigadores, instituciones públicas y privadas.

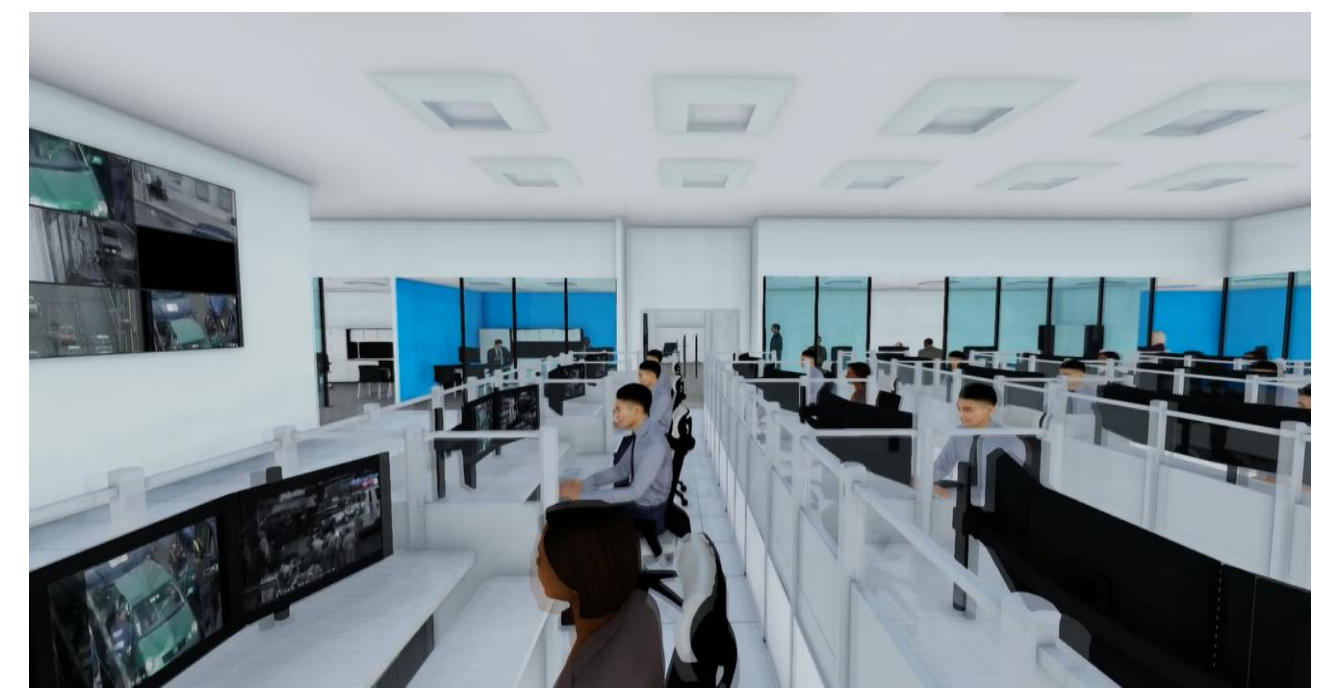

Fig. 03 Render interno de la plataforma de monitoreo. Elaboración propia.

Como ya se ha mencionado esta plataforma tendra diversos procesos como son:

\subsubsection{Generación de información}




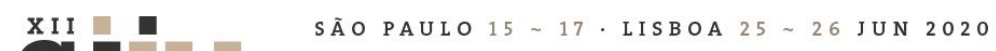

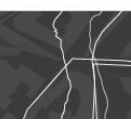

Que se realizará por medio de todas aquellas redes de monitoreo instaladas en el territorio, sistemas de alerta temprana, sociedad civil, organismos de respuesta, instituciones públicas y privadas comunidad científica, ONG y academia.

Toda la información que se recopile desde cada una de las fuentes debe de ser verificada antes de ejecutar acciones ya sea de respuesta operativa o análisis de escenarios prospectivos para la reducción de riesgos territoriales.

\subsubsection{Gestión operativa del territorio}

Articula las acciones de respuesta interinstitucional antes, durante y después de una emergencia o incidente. Realiza el monitoreo y seguimiento continuo de las redes de información tecnológica, así como, los eventos adversos y el control de territorio. En esta área se controlará el territorio, se monitoreara todos los sistemas urbanos, ejecutaran los planes emergentes y operativos, generaran mapas temáticos de estado actual y escenarios de emergencia, también se brindara atención a emergencias e incidentes. Teniendo una base de datos que permita planificar el territorio

\subsubsection{Gestión de conocimiento}

Analiza la información que se ha generado desde el área de gestión operativa y de las fuentes directas de información. La representación se la realiza por medio de escenarios, que son utilizados en la planificación para la reducción de riesgos de desastres.

- Genera escenarios prospectivos.

- Análisis de la información.

- Análisis de los indicadores territoriales.

- Alerta a planificación sobre las acciones de mitigación y adaptación.

\subsubsection{Planificación territorial y reducción de riesgos de desastres}

Analiza la información que se ha generado desde el área de gestión del conocimiento y otras fuentes directas para la reducción de riesgos de desastres. Busca organizar el territorio y cada uno de sus sistemas reduciendo en lo posible las vulnerabilidades, fomentando un desarrollo socioeconómico equilibrado, protegiendo el medio, preservando sus recursos, y mejorado la calidad de vida de la población.

Elabora propuestas de: planes de ordenamiento territorial, planes de uso de suelo, planes maestros, planes parciales, planes especiales y establece indicadores, procedimientos, metodologías y propuestas de ordenanzas que serán analizadas por la entidad competente en cada territorio para garantizar su aplicabilidad.

A continuación se detalla un modelo funcional, donde se aprecia la relación de los diferentes actores dentro de la plataforma y el vínculo que tienen con los diferentes procesos que se realizarán en la misma: 



\section{XII $\square$ SÃOPAULO15 17 LISBOA $25 \sim 26$ JUN 2020

contaminación de aguas, aumento de migración hacia las ciudades, asentamientos irregulares en áreas de riesgo, sobreexplotación de los recursos que en situaciones extremas desencadenan afectaciones severas, mismas que se agudizan por procesos inadecuados de gestión, necesitando las ciudades de un análisis multidisciplinario que cree ciudades resilientes en un espacio que preste condiciones de seguridad y sostenibilidad aceptables.

La República del Ecuador se encuentra circunscrita en una zona de riesgo debido a sus características geográficas, los datos y enseñanza que han dejado cada evento debe de ser utilizada para la generación de conocimiento y creación de territorios más resilientes, lamentablemente en la mayoría de las ciudades no se tiene conocimiento de que pasos seguir y se invierte más en reconstrucción que en construcción, por lo que la clave para formar ciudades resilientes se centra en una adecuada planificación territorial donde la prioridad debe ser la sociedad, siendo necesario la generación de información, la gestión operativa del territorio y la gestión de conocimiento que nos permita analizar el contexto territorial, crear escenarios, y poder ejecutar planes sostenibles que creen un nuevo paradigma colaborativo superando las carreras institucionales mediante una plataforma territorial tecnológica multinstitucional y multidisciplinaria .

\section{Referencias bibliográficas}

\subsubsection{Obra completa}

Carcedo, F. J. A. \& Cantos, J. O. (2002). Riesgos naturales. Reino de España: Ariel Ciencia.

Castro, M. Y Fernández, L. (2008) Informe de análisis de base de datos de pérdidas por desastres. Informe para PREDECAN, consultoría 025 de 2006 "Creación y/o actualización de inventarios históricos de desastres a nivel nacional en los cinco países de la Subregión Andina para el periodo de 1970 - 2006".

Centre for Research on the Epidemiology of Disasters (2018). Natural disasters. Bruselas: Autor

Comsión Europea y Comunidad Andina (2008), Pérdidas por desastres de impacto extremo, grande y menor en Ecuador, 1970 - 2007 Corporación OSSo. República del Ecuador: Autor.

Instituto Geográfico Militar de la República del Ecuador. (2013). Atlas Geográfico de la República del Ecuador. (1를. ed.) República del Ecuador: Autor.

López, d. S. O. (2017). Atributos ambientales para la evaluación de la vulnerabilidad frente a variabilidad y cambio climático en interfases rur-urbanas.

Maskerey, Adrew. (1993). Los desastres no son naturales. República de Colombia: Tercer Mundo.

Maskrey, A., Cardona, O., García, V., Lavell, A., Macías, J. M., Romero, G., \& Chaux, G. W. (1993). Los desastres no son naturales.

Paton, D., \& Johnston, D. (2017). Disaster resilience: an integrated approach. Charles C Thomas Publisher.

Real Academia de la Lengua. (2016). Diccionario. Reino de España: Real Academia de la Lengua.

Sepúlveda, S. (1998). Metodología para estimar el nivel de desarrollo sostenible en espacios territoriales (No. 4). IICA.

\subsubsection{Informes nacionales}

Comité de Reconstrucción. (2016). Informe s/n. República del Ecuador 


\section{SÃOPAULO15 17 LISBOA $25 \sim 26$ JUN 2020}

Seminário Internacional de Investigação em Urbanismo

Seminario Internacional de Investigación en Urbanismo

Secretaria de Gestión de Riesgo. (2016). Informe de situación n65. República del Ecuador

\subsubsection{Revistas}

Arteaga, C. \& Ugarte, A. M. (2015). Identidades en emergencia: la otra cara de la reconstrucción. El caso de Chaitén. Magallania, 43(3), 107-123.

Barton, J. R. (2009). Adaptación al cambio climático en la planificación de ciudades-regiones. Revista de Geografía Norte Grande, (43), 5-30.

Cutter, S. L., Burton, C. G. \& Emrich, C. T. (2010). Disaster resilience indicators for benchmarking baseline conditions. Journal of homeland security and emergency management, 7(1).

Hamui-Sutton, A., \& Varela-Ruiz, M. (2013). La técnica de grupos focales. Investigación en educación médica, 2(5), 55-60.

Lampis, A. (2013). Vulnerabilidad y adaptación al cambio climático: debates acerca del concepto de vulnerabilidad y su medición. Cuadernos de Geografía-Revista Colombiana de Geografía, 22(2), 17-33.

Méndez, R. (2012). Ciudades y metáforas: sobre el concepto de resiliencia urbana. Ciudad y Territorio Estudios Territoriales, 44(172), 216-217.

Miranda, F. M., \& Cornejo, F. (2010). El estudio de sistemas de ciudades. Una aproximación desde métodos de análisis de redes sociales. Tiempo y Espacio, (25).

\subsubsection{Fuentes electrónicas}

https://news.un.org/es/story/2019/09/1462322 (Consulta: 15/11/2019). 